\title{
UNIFORMIZABLE CAUCHY SPACES
}

\section{EVA LOWEN-COLEBUNDERS}

\author{
Vrije Universiteit Erussel \\ Depertement Wiskunde, F7 \\ Pleinlaan 2, 1050 Brussel \\ (Recelved April 13, 1981)
}

\begin{abstract}
ABSTRACI. A family $C$ of filters on a set $x$ is uniformizable if there is a uniformity on $X$ such that $C$ is its collection of Cauchy filters. Using the theory of completions and Cauchy continuous maps for Cauchy spaces, we obtain characterizations of uniformizable Cauchy spaces. In partizular, given a Cauchy structure $C$ on $X$ we investigate under what conditions the filter u(C)= $\cap F \times F$ is a uniformity and $C$ is $F \in C$

its collection of cauchy filters. This problem is treated using Cauchy covering systems.

KEY WORDS AND PHRASES. Uniformity, Cauchy space, completion, cauchy continuous map, realcompactness, Cauchy covering system.

1980 MATHEMATICS SUBJECT CLASSIFICATION CODES. $54 A 20,54 E 15$.
\end{abstract}

1. INTRODUCTION.

As background for reading this paper, the methematician unfamiliar with Cauchy spaces might consult the paper "Completions of uniform convergence spaces" by E. Reed [1].

A Cauchy structure $C$ on a set $X$ is a collection of filters on $x$ satisfying the conditions that for every point $x$ of $x$ the filter gene- 
rated ty $\{x\}$ belongs to $C$. If a filter belongs to $C$, then so does every finer filter and, if two filters in $C$ have a supremum, their intersection belongs to $C$. In [2] Keller has shown that these properties are necessary and sufficient in order to have a uniform convergence structure on $X$ in the sense of [3] such that $C$ is exactly the collection of its Cauchy filters. If there exists a uniform structure in the sense of [4] with $C$ as its family of Cauchy filters, $C$ is said to be uniformizable. This notion was introduced in [5]. The main purpose of this paper is to find supplementary conditions on a Cauchy space in order to characterize the uniformizability. When $C$ is the collection of convergent filters of a topological space, the problem reduces to the complete uniformizability of the topology. Two different attempts will be made in studying the uniformizability. The first method uses Cauchy continuous maps. Barring measurable cardinals, we obtain that a Cauchy space $(x, C)$ is uniformizable if and only if there exists a collection of realvalued Cauchy continuous maps such that $C$ is the family of all filters having a Cauchy image on $\mathbb{R}$ for every map in the collection.

A second method uses completions. We show that $(X, C)$ is uniformizable if and only if there is a dense Cauchy embedding in the collection of convergent filters of a completely uniformizable topological space, more specifically if and only if its kowalsky or ultrafilter completion [1] is a completely uniformizable topological space. Barring measurable cardinals the previous condition is equivalent to the realcompactness of the completion. From this property some conditions on $C$ can be derived.

Any uniformity on $x$ having $C$ as its family of cauchy filters is coarser than the filter $u(C)=\underset{F \in C}{n} F \times F$. When $C$ is the collection of convergent filters of a topological space, then u(C) is the neighborhood filter of the diagonal. In the second part of the paper we shall 
investigate if $u(C)$ is a uniformity and if so, under what conditions C is its collection of Cauchy filters. A very useful notion to treat these problems is that of a Cauchy covering system, a notion introduced in [6] and extending the definition of covering system of a convergence space [7]. Using Cauchy covering systems we characterize the property of $u(C)$ being a uniformity. We show that $u(C)$ has $C$ as its family of Cauchy filters if and only if $C$ is uniformizable. Extending the notion of paracompactness we introduce Cauchy paracompactness for Cauchy spaces. We show that for every Cauchy space with minimum elements in its equivalence classes, which is Cauchy paracompact and round, $u(C)$ is a uniformity with $C$ as its family of Cauchy filters. In the final section we treat the special case of a totally bounded Cauchy space.

\section{PRELIMINARIES}

We recall some of the fundamental definitions on Cauchy spaces needed in the paper. For additional information, we refer to [1] [2] [6] [8] [9] or [10]. Let $x$ be a set and $F(x)$ the collection of f11ters on $x$. A subset $C \subset F(x)$ is a Cauchy structure if.

(i) For every $x \in X$ the filter $\dot{x}$ generated by $\{x\}$ belongs to $C$ (ii) If $F \in C$ and $G \supset F$ then $G \in C$

(iii) If $F \in C$ and $G \in C$ and $F \vee G$ exists then $F \cap G \in C$ The filters in $C$ are Cauchy filters and the space $(X, C)$ a Cauchy space. Every uniform space $(x, U)$ in the sense of [4] induces a cauchy structure c(U) which is its collection of Cauchy filters. A Cauchy structure induces a convergence structure: $F \rightarrow x$ if and only if $F \cap \dot{x} \in C$. The Hausdorff property, closure operation, and continuity for a Cauchy space are defined in the induced convergence space. Throughout the paper the Cauchy space $(X, C)$ used in the formulation of the theorems is supposed to be Hausdorff. A map $f$ between two Cauchy spaces $(X, C)$ and $(Y, D)$ is Cauchy continuous if the image $f(F)$ of overy $F$ in $C$ 
belongs to $D$. Here $f(F)$ is the filter generated ty $\{f(F) \mid F \in F\}$. Cauchy continuous maps are continuous. Cauchy sutspaces, Cauchy embeddings, and Cauchy homeomorphisms are defined in the obvious way. For Cauchy continuity of maps between uniform spaces, we use the induced Cauchy spaces. When $C$ and $D$ are Cauchy structures on the same set $x$, then $C<D$ ( $C$ is coarser, $D$ is finer) if and only if the identity fromi $(x, D)$ to $(x, C)$ is Cauchy continuous. A Cauchy structure is totally bounded if every ultrafilter is Cauchy; it is complete if every Cauchy filter converges. For every convergence sface the collection of its convergent filters is a complete cauchy space. A completion. $(Y, D, K)$ of a Cauchy space $(X, C)$ consists of a complete Cauchy space $(Y, D)$ and a Cauchy embedding $k: X \rightarrow Y$ such that $k(X)$ is dense in $Y$. Two filters in $C$ are equivalent if their intersection belongs to $C$. For a filter $F$ in $C$, let $\langle F\rangle$ be its equivalence class. $X^{*}$ is the set of all equivalence classes and $j: x \rightarrow x^{*} \operatorname{maps} x$ to $\langle\dot{x}\rangle$. In [ 1], [5], [6], [8], [9], [10], methods are given to construct completions. The Kowalsky completion will be used explicitely in the text. Let $\Sigma^{*}$ be the collection of all maps $\sigma: x^{*} \rightarrow F(x)$ such that $\sigma(p) \in p$ for $p \in x^{*}$ and let $\Sigma=\left\{\sigma \in \Sigma^{*} \mid \sigma(j(x))=\dot{x}\right.$ for $\left.x \in x\right\}$. For each $\sigma \in \Sigma$ and $A C x, \operatorname{let} A^{\sigma}=\left\{p \in x^{*} \mid A \in \sigma(p)\right\}$. For a filter $F$ on $x$, let $F^{\sigma}$ be the filter on $x^{*}$ generated ty $\left\{F^{\sigma} \mid F \in F\right\}$. A corvergence structure on $x^{*}$ is defined as follows : A filter $\psi$ on $x^{*}$ converges to some $p \in x^{*}$ If and only if, for every $\sigma \in \Sigma$, there is a filter $F \in p$ such that $F^{\sigma} \cap \dot{p} \subset \psi$. Finally $c^{*}$ is the collection of all convergent filters on $x^{*} \cdot\left(x^{*}, C^{*}, j\right)$ is the Kowalsky completion of $(x, C)$. For $\sigma \in \Sigma^{*}$ and $A$ and $B$ subsets of $x$, we have the relation $A<\sigma B$ if and only if $A \subset B$ and, for every $p \in x^{*}$, either $B$ or $x \mid A$ belongs to $\sigma(p)$. For $F \in F(x)$, let $r_{\sigma}(F)$ be the filter $\left\{A \mid F<_{\sigma} A\right.$ for some $\left.F \in F\right\}$. A Cauchy space $(x, C)$ is called round if it is closed for the operation $r$ for every $\sigma \in \Sigma^{*}$. 


\section{WEAK CAUCHY STRUCTURES}

Let $\phi=\left\{f_{i} \mid i \in I\right\}$ be a collection of maps where $f_{i}: X \rightarrow\left(Y_{i}, D_{i}\right)$ for $i \in I$. Let $F \in C$ if and only if $f_{i}(F) \in D_{i}$ for every $i \in I$. This cefines a Cauchy structure on $x$. (not necessarily Hausdorff). called the weak Cauchy structure of $\phi$. It is the coarsest Cauchy structure on $x$ making all the mops in $\phi$ Cauchy continuous. For a Cauchy space $(x, C)$, let $\Gamma_{u}(x, C)$ be the collection of all Cauchy continuaus maps from $(x, C)$ to uniform spaces and $\Gamma_{\mathbb{R}}(x, C)$ the collection of all realvalued Cauchy continuous maps on $(x, C)$.

THEOREM 3,1. A Cauchy space $(x, \mathcal{C})$ is uniformizable if and only if one of the following equivalent conditions are fulfilled:

(1) There exists a nonempty collection $\phi \subset \Gamma_{u}(x, C)$ such trat $C$ is the weak structure of $\phi$.

(2) $C$ is the weak structure of $\Gamma_{u}(x, \mathcal{C})$.

PFoof. If $C$ is uniformizable and $U$ is a uniformity on $x$ such that $c(U)=C$, consider $i$ the identity from $(x, C)$ to $(x, U)$ and let $\phi=\{i\}$. Hence (1) is satisfied.

To shcw that (1) implies (2), let $\phi \subset \Gamma_{u}(x, \mathcal{C})$ such that $C$ is the weak Cauchy structure of $\phi$. Fhen $C$ is finer than the wak structure of $\Gamma_{u}(x, \mathcal{C})$ and the latter is finer than the wak structure of $\phi$ which is $C$. Hence, they all coincide and (2) is fulfilled, To complete the proof, suppose $C$ is the weak Cauchy structure of $\Gamma_{u}(x, C)$. Let $u$ be the weak uniformity of $\Gamma_{u}(x, C)$, which is the coarsest uniformity on $x$ making all the maps in $\Gamma_{U}(x, C)$ uniformly cantinuous. Then we have $c(U)=C$ and $C$ is uniformizable.

ThEOREM 3.2. If a Cauchy spaee $(X, C)$ is uniformizable, the weak uniformity of $\Gamma_{U}(x, C)$ is the finest uniformity with $C$ as 1 ts family of Cauchy filters and among the uniformitios with $C$ as family of Cauchy filters it is the only uniformity making all maps in $\Gamma_{u}(x, C)$ uniformly continuous. 
PROOF. Let $U$ be the weak uniformity of $\Gamma_{u}(x, C)$. In the proof of the previous theorem, it is shown that $c(U)=C$. Let $W$ be another uniformity on $x$ such that $c(w)=C$. Then the identity from $(x, C)$ to $(x, W)$ belongs to $\Gamma_{u}(x, C)$. Hence $W \subset U$. If $W$ in addition is supposed to make all the maps in $\Gamma_{u}(x, C)$ uniformly continuous, then $w=U$ since $U$ is the coarsest such structure.

In the next theorem, we suppose $x$ has non measurable cardinality. which is a very light restriction to make since no measurable cardinals are known. With this restriction complete uniformizability of a topological space is equivalent to realcompactness [11].

THEOREM 3.3. A Cauchy space $(X, C)$ is uniformizable if and only if one of the following equivalent conditions is fulfilled:

(1) There exists a nonempty collection $\phi \subset \Gamma_{\mathbb{R}}(x, C)$ such that $C$ is the weak Cauchy structure of $\phi$.

(2) C is the weak Cauchy structure of $\Gamma_{\mathbb{R}}(x, C)$.

PROOF. Let $U$ be a uniformity on $x$ such that $c(U)=C$. Suppose $F$ is a filter on $X$ such that for every $f \in \Gamma_{\mathbb{R}}(x, C)$ we have $f(F)$ is IR-Cauchy. Let $\left(x^{*}, u^{*}\right)$ be the completion of $(x, u)$, j the embedding of $x$ in $x^{*}$, and $C_{\operatorname{IR}}\left(x^{*}, u^{x}\right)$ the collection of all real valued continuous maps on $\left(x^{*}, u^{*}\right)$. It follows that hoj(F) is IR-Cauchy for every $n \in C_{I R}\left(x^{x}, u^{*}\right)$. But then $j(F)$ is a Cauchy filter for the weak uniformity of $C_{\text {IR }}\left(x^{*}, u^{*}\right)$ on $x^{*}$. Since we are barring measurable cardinals, this uniformity is complete and so $f(F)$ converges. Hence $F \in C$. The other implications in the theorem follow trivially from $(3.2)$.

\section{COMPLETIONS}

In this paragraph we investigate the relation between the uniformizability of $(X, C)$ and properties of its completions.

THEOREM 4.1. A Cauchy space $(X, C)$ is uniformizoble if and only if it is embedded in a (dense) Cauchy subspace of a completely uniformizable topological space. 
PROOF. If $(x, C)$ is uniformizable by $u$, then the completion $\left(x^{x}, u^{x}\right)$ provides a completely uniformizable topological space in which $(X, C)$ is densely Cauchy embedded.

If there is a Cauchy embedding $j$ of $(X, C)$ into a topological space $Y$ having a complete uniformity $w$, then $j^{-1} \times j^{-1}\left(a^{\prime}\right)$ is a uniformity on $X$ for which $C$ is the collection of Cauchy filters.

In [1] several methods are given to construct completions of a given Cauchy space. Some of them preserve uniformizability, for example the Kowalsky completion and the ultrafilter completion. In fact, when $(X, C)$ is uniformizable, these completions are Cauchy homeomorphic to the Cauchy structure of the Bourbaki completion. From the previous theorem, we now immediately have the following property. THEOREM 4.2. A Cauchy space is uniformizable if and only if its Kowalsky completion (ultrafilter completion) is a completely uniformizable topological space.

We will apply this theorem for the case of the Kowalsky completion in order to find explicit conditions for uniformizability. A fundamental property that will be needed is the regular extension property [1]. It means that every Cauchy continuous map from a Cauchy space $(x, C)$ to a complete and regular Cauchy space has a unique (Cauchy) continuous extension to the Kowalsky completion. In the special case of $\mathbb{R}$ this implies that there is a bijection $x$ from $\Gamma_{\mathbb{I}}(x, C)$ to $\Gamma_{\text {IR }}\left(x^{*}, c^{*}\right)$ mapping $f$ to its extension $f^{*}$. This bijection, in fact, is a ring isomorphism and a lattice isomorphism. For $f \in \Gamma_{I P}(x, C)$ and $p \in x^{*}$, the value of $f^{*}$ in $p$ is the limit of $f(F)$ where $F$ is a Cauchy filter arbitrarily chosen in $p$. This limit is unique and equals

$\cap \overline{f(F)}$. We shortly write lim $f(F)$. $F \in F$

THEOREM 4.3. For nonmeasurable cardinals, a Cauchy space $(X, C)$ is uniformizable if and only if each of the following conditions (1), (2), (3) and (4) hold. Condition (4) can be replaced by the equivalent 
condition (4').

(1) Every equivalence class in $C$ has a minimum element.

(2) The minimal Cauchy filters have an open base.

(3) For every minimal Cauchy filter $M$ and for every $M \in M$ there exists a Cauchy continuous map $f$ such that $11 \mathrm{~m} f(M)=1$ and $\lim f(F)=0$ for every Cauchy filter containing $x \backslash M$.

(4) In the collection $\zeta(x, C)=\left\{\zeta(f) \mid f \in \Gamma_{I R}(x, C)\right\}$ where $\zeta(f)$ is the subset of $X^{*}$ consisting of those $p$ for which there exists on $F \in p$ with lim $f(F)=0$, every subcollection, maximal for the finite intersection property and with the countable intersection property, has a nonempty intersection.

(4') Let $\left(r_{f}\right)_{f \in \Gamma_{\mathbb{R}}}(x, C)$ be a collection of real numbers indexed by $\Gamma_{I F}(x, C)$. If for every finite subset $I_{0} \subset \Gamma_{\mathbb{I R}_{R}}(x, C)$ and for every $\varepsilon>0$ there is an $x \in X$ such that $\left|f(x)-r_{f}\right|<\varepsilon$ for every $f \in I_{0}$. then there is a Cauchy filter F such that $r_{f}=11 m f(F)$ for every $f \in \Gamma_{\mathbb{R}}(x, C)$.

PROOF. Suppose $(X, C)$ is uniformizable by a uniformity $U$. Then (1) and (2) hold. Let $\mu$ be the map on $x^{*}$ to $F(x)$ which maps $f(x)$ to $\dot{x}$ and $p \in x^{x} \backslash j(x)$ to the minimum element in $p$. Then, for $p \in x^{x}$, the neighborhoodfilter in the topology $T^{*}$ of the completion is the filter $M^{\mu}$ where $M$ is the minimum in $p$. Condition (3) then follows immediately from the complete regularity of this topology. For $f \in \Gamma_{\mathbb{R}}(x, C)$, we have $\zeta(f)=f^{x-1}(0)$. so $\zeta(x, c)$ is the collection of zerosets for $C_{\mathbb{R}}\left(x^{x}, T^{*}\right)$, the collection of realvalued continuous maps on $\left(x^{*}, T^{*}\right)$. Condition (4) is nothing but the realcompactness of $T^{*}$. In order to prove $\left(4^{\circ}\right)$, let $\pi$ be the product of real lines indexed by $\Gamma_{\mathbb{R}}(x, c)$, let $\rho:(x, c) \rightarrow \Pi$ be the map $\rho(x)=(f(x)) f_{f \in \Gamma_{\mathbb{R}}(x, c)}$ for $x \in x$, and let $\sigma:\left(x^{*}, c^{*}\right) \rightarrow \Pi$ be the $\operatorname{map} \sigma(p)=\left(f^{*}(p)\right)_{f \in \Gamma_{\mathbb{R}}}(x, c)$ for $p \in x^{*}$. Then $\sigma$ is the extension of $\rho$. Since $\left(x^{*}, T^{*}\right)$ is realcompact, the map $\sigma$ is a homeomorphism onto $\sigma\left(x^{*}\right)$ which is closed in $\pi$. 
Hence $\sigma\left(x^{*}\right) \supset \overline{p(x)}$. Condition (4') follows.

Suppose conditions (1), (2). (3). (4) are fulfilled. We show that the Kowalsky completion is a completaly regular realcompact topologlcal space. Lat $\mu$ be tha map as defined above. Then $\mu$ belongs to $\sum$. It is clear that a filter converges to p for the kowalsky structure If and only if it is finer than $M^{\mu}$ whore $M$ is the minimum of $P$. If $M$ 1s opan, so is $\mathrm{M}^{\mu}$. It follows that the kowalsky completion $1 \mathrm{~s}$ topological and (3) and (4) clearly imply that it is completely regular and realcompact. If $\left(4^{\prime}\right)$ ia fulfiliad instaad of (4), then, since $\left(x^{*}, c^{*}\right)$ is completely regular. We have that the map o defined above Is a homeomorphism onto $\sigma\left(x^{*}\right)$. It follows from (4') and from the continuity of $\sigma$ that $\sigma\left(x^{x}\right)=\overline{p(\bar{x})}$. So $\sigma$ is closed and $\left(x^{x}, c^{x}\right)$ is raalcompact.

\section{CAUCHY COVERING SYSTEMS AND THE FILTER U(C)}

For a Cauchy $\operatorname{space}(X, C)$, lat $u(C)=n\{F \times F \mid F \in C\}$. u(C) clearly is a filter with a symmetric base and whose elements all contain the digonal. In this section. we investigate under what conditions u(C) is a uniformity and, if so, whether C is, its collection of Cauchy filtars, 1.e. $c(u(C))=C$.

When C is the collection of convergent filters of a topological space, $U(C)$ is the nelghborhood filter of the diagonal in $x x$. There oxist completely uniformizable topological spaces which are not normal [11]. The nelghborhood filter of the diagonal in such spaces is not a uniformity. Hence the uniformizability of $C$ does not imply that $u(C)$ is a uniformity.

Conversely for the open ordinal space $(0 . \Omega)$ f. 1 . . the nelghborhood filter of the diggonal is a unique uniformity and it is non complete [12]. Hence if $u(C)$ is a uniformity $C$ nesd not be uniformizable. It is evident that when $u(C)$ is a uniformity wo always have $C C C(u(C))$. THEUREM 5.1. $u(C)$ is a uniformity if and only if it is the waak 
uniformity of $\Gamma_{u}(x, C)$.

Proof. The if part is trivial. For the only if part, suppose $u(C)$ is a uniformity. Then the identity from $(x, C)$ to $(x, u(C))$ is Cauchy continuous. So $u(C)$ is coarser than the weak uniformity of $\Gamma_{u}(x, C)$. On the other hand. let $f$ be a Cauchy continuous map from $(x, C)$ to a uniform space $(Y, W)$. For $W \in W$ and $F \in C$, choose $F \in F$ such that $f(F) \times f(F) \subset W$. Then, for $V=\underset{F \in C}{U} F \times F$, we have $f x f(V) \subset W$. It follows that $u(C)$ makes $f$ uniformly continuous and so $u(C)$ equals the weak uniformity of $\Gamma_{u}(x, C)$.

From (3.2) and (5.1) we now have the following property.

COROLLARY 5.2. If $(X, C)$ is a Cauchy space and if $u(C)$ is a uniformity, then we have $c(u(C))=C$ if and only if $C$ is uniformizable.

If the kowalsky completion $\left(x^{x}, c^{x}\right)$ of $(x, C)$ is divisible, which means that the nelghborhood filter $V\left(\Delta^{*}\right)$ of the diagonal in $x^{x} \times x^{x}$ 1s aniformity, and if $\left(x^{*}, c^{*}\right)$ is completely uniformizable, then $u(C)$ is the trace of $V\left(\Delta^{*}\right)$ on $x \times x$, and so it is a uniformity on $x$ with $c(u(C))=C$. In order to prove the converse of this statement. we make a preliminary assumption on $\left(x^{*}, c^{*}\right)$, namely that the neighborhoods of the diagonal in $x^{*} x . x^{*}$ have a closed base. In [13] this property is called $\Delta$-normality and the precise position of $\Delta$-normality with regard to normality conditions and other separation axioms is settled there.

THEOREM 5.3. If $u(C)$ is a uniformity and $C$ is uniformizable, then $\left(x^{*}, C^{*}\right)$ is divisible if and only if $\left(x^{*}, C^{*}\right)$ is $\Delta$-normal.

PROOF. If $\left(x^{*}, c^{*}\right)$ is divisible then it clearly is $\Delta$-normal. To prove the converse suppose $\left(x^{*}, C^{*}\right)$ is $\Delta$-normal, u(C) is a uniformity, and $C$ is uniformizable. Let $V$ be a closed neighborhood of the diagonal of $x^{*} \times x^{*}$. From theorem 4.3 1t follows that for every $p \in x^{*}$ we can choose an $M_{p} \in M_{p}$ such that, (with the notations of 4.3). $M_{p}^{\mu} \times M_{p}^{\mu} \subset v$. Then we have $\underset{p \in X^{*}}{U} M_{p} \times M_{p} \in U(C)$ and so 
$\left.{ }^{c 1} x_{x x^{*}}{ }_{p \in x^{*}}^{U} M_{p} \times M_{p}\right) \subset V$. But then $V\left(\Delta^{*}\right)$ is the uniformity $(u(C))^{*}$ of the completion of $(x, u(C))$.

DEFINITION 5.4. A collection of subsets of a Cauchy space $(X, C)$ is a Cauchy covering system if every filter in C contains a member of the collection: it is a basic Cauchy covering system if for every filter in C there are a finite number of members of the collection whose union belongs to the filter.

The first notion was introduced in [6]. Both definitions in (5.3) extend the notions of covering system and basic covering system of a convergence space introduced in [7]. The condition in the following theorem extends the characterization by means of open covers given in [14] of the property that the neighborhood filter of the diagonal of $x \times x$ is a uniformity. The proof is straightforward.

THEOREM 5.5. u(C) is a uniformity if and only if, for every Cauchy covering system $A=\left\{A_{1} \mid 1 \in I\right\}$, there exists a Cauchy covering system $B=\left\{B_{j} \mid j \in J\right\}$ such that, if $B_{j} \cap E_{k} \neq \phi$ and if $x$ and $y$ belong to $B_{j} \cup B_{k}$, there 1 s an $1 \in I$ such that $x$ and $y$ both belong to $A_{1}$.

In order to prove an extension of the theorem that every paracompact space is completely uniformizable by the neighborhood filter of the diagonal, we need some preliminary definitions.

DEFINITION 5.6. A Cauchy covering system of a Cauchy spoce is Cauchy finite if every Cauchy filter contains a set intersecting only finitely many members of the system.

DEFINITION 5.7. A Cauchy space $(X, C)$ is (basic) Cauchy paracompact If for every Cauchy covering system, there is o (basic) Cauchy covering Cauchy finite refinement.

THEOREM 5.8. If $(X, C)$ is a (basic) Cauchy paracompact space which is round and has a minimum Cauchy filter in each equivalence class, then $u(C)$ is a uniformity and $c(u(C))=C$. 
PRodf. Let $U \in U(C)$. For every mintmal Cauchy filter $M$ we choose an $A \in M$ sueh that $A \times A C U$. Let $A$ be the collection of sets $A$ constructed in this way. Lat $\lambda$ be the map on $x^{x}$ to $F(x)$ which maps p to ita minimum element $\lambda(B)$. Sinee C is round, we have $r_{\lambda}(M)=M$ for every minimal lement $M$ in $C$. We make a new Cauchy cover $A$ ' in the following way. For every minimal Cauchy filter $M$ and for $A \in A \cap M$. we choose $A^{\prime} \in M$ such that $A^{\prime}{ }_{\lambda} A$. This Cauchy covering syster. has a (bagio) Cauchy covering Cavehy finite refinement $B$. For $B \in B$. we chooge $A_{B} \in A$ such that $B<\lambda A_{B}$ and we let $V_{B}=A_{B} \times A_{B} U\left(x \mid\right.$ 目) $\times(x \mid B)$. Then $V_{B}$ belongs to $u(C)$. Let $V=\underset{\theta \in B}{n} V_{B}$ and let $M$ ba a minimal Cauchy filter. There exists an $M E M$ intersecting on ly finitely many members of $B$. We have $M \times M \subset \cap\left\{V_{\text {目 }} \mid \mathrm{g} \in \boldsymbol{B}\right.$. 目 $\left.\cap M=\phi\right\}$. It follows that $V \in u(C)$. For $x \in X$, the filter $\dot{x} \in C$ and so there exists a $B \in B$ containing $x$. But than $V(x) \subset V_{B}(x) \subset A_{B}$ and so $V(x) x V(x) \subset U$. Since $V$ is symmetrie we have $V \cdot V \subset U$,

It remaing to be hown that $e(u(C)) C C$. Let $F \in c(u(C))$. Suppose $F M$ for avery minimal Cauchy filter $M$ in $C$. For every $M$, choose $M \in M, M \notin F$, and let $A$ be the cauchy covering system consigting of the sots $M$ constructed in this way. In the first part of the proof, It Is sown that a $V \in u(C)$ axists such that $(V(x))_{x} \in$ refines $^{A}$. Choose $F_{0} \in F$ weh that $F_{0} \times F_{0} \subset V$ and choose $x_{0} \in F_{0}$. There 18 an $M_{0}$ and an $M_{0} \in M_{0} \cap A$ with $V\left(x_{0}\right) \subset M_{0}$ and $F_{0} \& M_{0}$. A contradiction fol10ws.

THEOREM 5.8. A Cauchy space ( $X, C)$ has minimum elements in each oquivalonce clasa, Is raund, and Cauchy paracompact if and only if the Kowalsky completion is a paracompact topological space.

PRoof. Fram (4.2) and (5.8). It follows that when C has minima, Is round, and Cauchy paracompact, $x^{x}$ is a topological spaca. Let $\mu$ be the map as in $(4.3)$, then $\left\{0^{\mu} \mid a C X\right.$, open\} is a base for the 
open sets of $x^{*}$. Let $\left\{0_{1} \mid 1 \in I\right\}$ be an open cover of $x^{*}$ with $0_{1} \subset x$ open. Then $\left\{0_{1} \mid i \in I\right\}$ is a Cauchy cover of $x$. Let $\left\{Q_{j} \mid j \in J\right\}$ be a Cauchy finite refinement. This collection can be chosen open (4.3). Then $\left\{Q_{j} \mid j \in J\right\}$ is an open locally finite refinement of the given collection.

Suppose $x^{*}$ is a paracompact topological space. Then $(x, C)$ has minima (4.3). If $A$ is a Cauchy cover of $X$ which may be chosen to be open, then $\left\{A^{\mu} \mid A \in A\right\}$ is an open cover of $X^{*}$. Let $B$ be a locally finite open refinement. Then the collection $\left\{f^{-1}(B) \mid B \in B\right\}$ is a Cauchy cover of $x$ which is a Cauchy finite refinement of the given collection. Since $x^{*}$ is paracompact and Hausdorff, it is regular. It follows from a corollary in [9] that the Kowalsky completion coincides with the completion constructed there. Therefore every minimal Cauchy filter $M$ satisfies $M^{\mu}=\sum M$ where $\Sigma M=\{\Sigma M \mid M \in M\}$ and $\sum M=\left\{p \in X^{*} \mid M \in F\right.$ for some $\left.F \in p\right\}$. On the other hand since $M$ has an open base, we have $M^{\mu}=M^{\lambda}$ where $M^{\lambda}$ is the filter generated by $\left\{M^{\lambda} \mid M \in M\right\}$ and $M^{\lambda}=\left\{p \in x^{*} \mid M \in \lambda(p)\right\}$ for the map $\lambda$ introduced in $(5.8)$. Finally it is easily checked that $M^{\lambda}=\sum M$ if and only if $r_{\lambda} M=M$. It follows that $X$ is round.

REMARK 5.10. If $u(C)$ is a uniformity and $c(u(C))=C$, then clearly $C$ has minima in its equivalence classes and $C$ is round. But C need not be Cauchy paracompact. This follows from a result of Corson [15], that a completely uniformizable topological space for which the neighborhoodfilter of the diagonal is a uniformity need not be paracompact.

In the final section we treat the special case of a totally bounded Cauchy space.

THEOREM 5.11. A totally bounded Cauchy space is basic Cauchy paracompact.

PRoOf. Let $A$ be a Cauchy cover in a totally bounded Cauchy space 
$(x, C)$. For every ultrafilter on $x$, there is an $A \in A \cap U$. It follows that there exist $A_{1} \ldots A_{n}$ in $A$ such that $\bigcup_{1=1}^{n} A_{1}=x$. Let $B=\left\{A_{1} \ldots A_{n}\right\}$; then this is a basic covering Cauchy finite refinement.

THEOREM 5.12. For a totally bounded Cauchy space the following properties are equivalent.

(1) C is uniformizable

(2) $x^{x}$ is a compact Hausdorff topological space

(3) C is round and has a minimum in each equivalence class

(4) $u(C)$ is a uniformity and $c(u(C))=C$

(5) There is exactly one uniformity with $C$ as its collection of Cauchy filters PROOF. $(1) \Rightarrow(2)[1] ;(2) \Rightarrow(3)(5.9),(3) \Rightarrow(4)(5.8)$ and (5.11); (4) $\Rightarrow(5)$ From (4) it follows that $x^{*}$ is compact Hausdorff and so it has a unique uniformity; (5) $\Rightarrow$ (1) trivial.

ACKNOWLEDGEMENT. I would like to thank Professor D.C. Kent for the many fruitful suggestions on the subject.

\section{REFERENCES}

1. REED, E. "Completions of uniform convergence spaces" Math. Ann. 194. 83-108, 1971 .

2. KELLER, H. "Die Limes-uniformisierbarke1t der Limesrăume" Math. Ann. 176. 334-341, 1968.

3. COOK, C. and FISCHER, H. "Uniform convergence structures" Math. Ann. 173, 290-306, 1967.

4. BOURbaKI, N. "Topologie générale" Eléments de Mathématique, Fas II chap. 1. Hermann, Paris, $4^{\text {th }}$ ed., 1965.

5. FRIC, R, and KENT, D. "On the natural completion functor for Cauchy spaces" Bull. Austral. Math. Soc. 18, 335-343, 1978.

6. GAZIK, R. and KENT, D. "Regular completions of Cauchy spaces via function algebras" Bull. Austral. Math. Soc. 11.77-88, 1974. 
7. FELDMAN, W. "Axioms of countability and the Algebra $C(x)$ " Pacific J. Math. 47. 81-89, 1973 .

8. FRIC, R. and KENT, D. "Completion functors for Cauchy spaces" Internat. J. Math. and Math. Sc1. 2,4, 589-604, 1979 .

9. KENT, D. and RICHARDSON, G. "Regular completions of Cauchy spaces" Pacific J. Math. 51, 483-490, 1974.

10. RAMALÉY, J. and WYLER, 0 . "Cauchy spaces I and II" Math. Ann. $187,175-199,1970$.

11. GILLMAN, L. and JERISON, M. "Rings of continuous functions" Springer-Verlag. $2^{\text {ńd }}$ ed.. 1976.

12. DIEUDONNE, J. "Un example d'espace normal non susceptible d'une structure uniforme d'espace complet" C.R. Acad. Sci. Paris 209, $145-147,1939$.

13. HART, K. "Spaces for which the diagonal has a closed neighborhoodbase" preprint.

14. COHEN, H. "Sur un problème de M. Dieudonné" C.R. Acad. Sci. Par1s 234. 290-292, 1952 .

15. CORSON, H. "Normality in subsets of Product spaces" Amer. J. Math. 81, 785-796, 1959. 


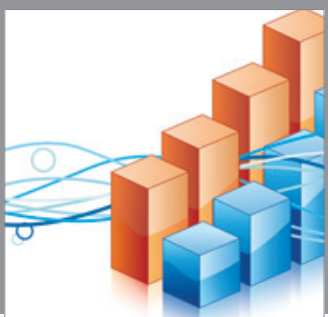

Advances in

Operations Research

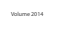

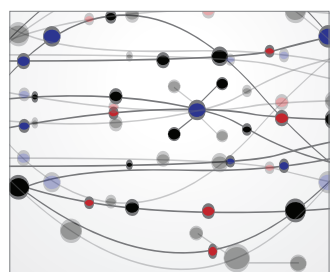

\section{The Scientific} World Journal
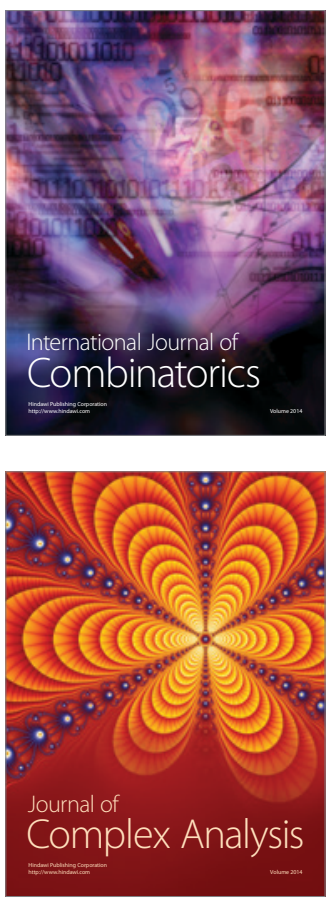

International Journal of

Mathematics and

Mathematical

Sciences
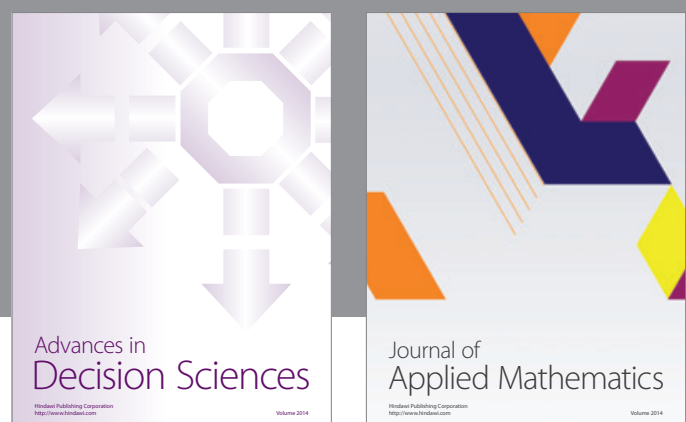

Journal of

Applied Mathematics
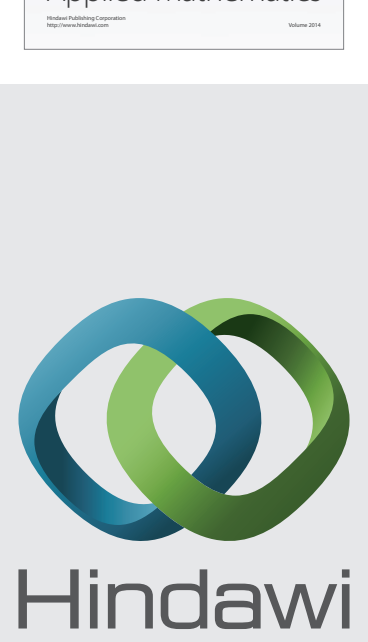

Submit your manuscripts at http://www.hindawi.com
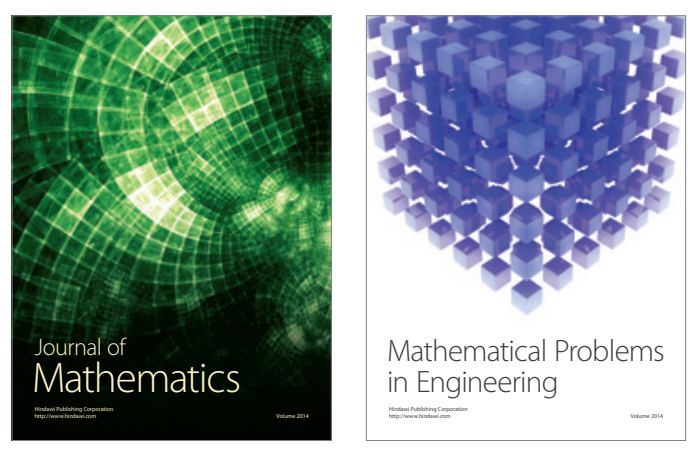

Mathematical Problems in Engineering
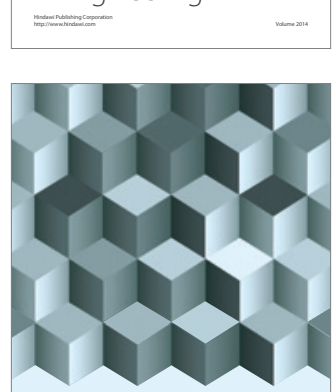

Journal of

Function Spaces
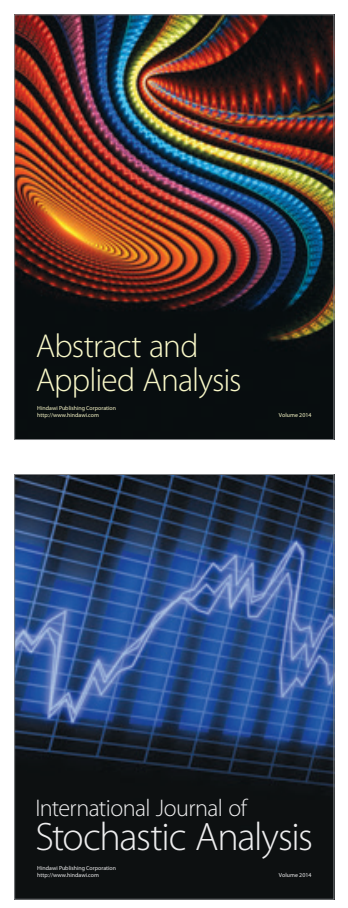

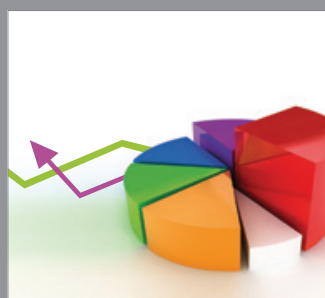

ournal of

Probability and Statistics

Promensencen
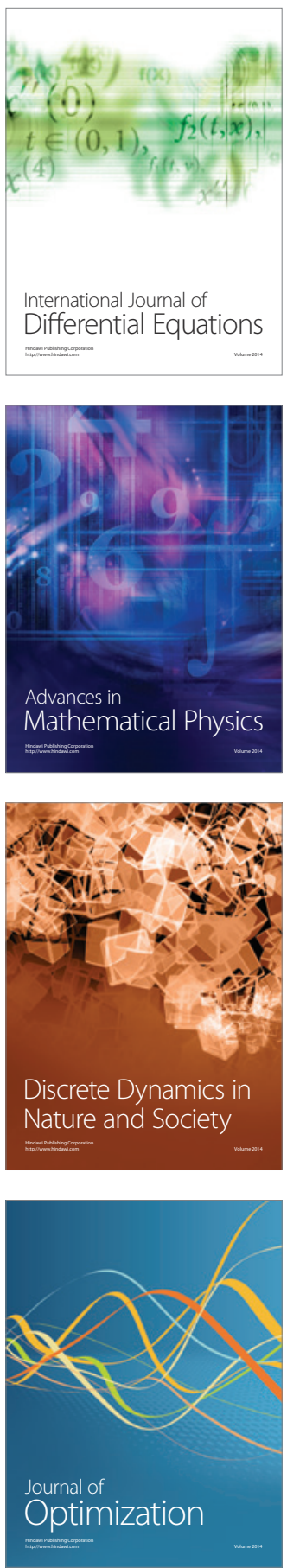